\title{
Risk assessment to guide prostate cancer screening decisions: a cost-effectiveness analysis
}

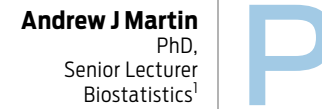

rostate cancer screening using the prostate-specific antigen (PSA) test has been available to asymptomatic men in Australia since the late 1980s; however, substantial uncertainty still remains over the net benefit of early detection.

Based on the trial evidence available to date, ${ }^{1}$ routine PSA screening for asymptomatic men is not recommended in Australia; ${ }^{2}$ however, access to PSA testing is available and publically subsidised in Australia through the Medicare Benefits Schedule. The Royal Australian College of General Practitioners (RACGP) recommends that general practitioners assist men seeking PSA screening to reach informed decisions by weighing the potential benefits of screening against the inconvenience and harms of biopsies resulting from false-positive PSA test results, as well as the side effects of unnecessary surgery and/or radiotherapy for indolent cancers that currently cannot be reliably distinguished from those of a more aggressive nature. $^{2}$

While debate continues about the interpretation of existing trial data in decisions about the PSA screening of asymptomatic men in general, there is a recognition that the trade-off between the potential benefits, harms and financial costs associated with PSA screening may theoretically be more favourable for a subgroup of asymptomatic men who are at elevated risk of prostate cancer. ${ }^{3-5}$ One reason for this is that the positive predictive value (PPV) of a test is greater in high-risk populations, because PPV is a function of not only the test's sensitivity and specificity but also of the prevalence of the condition of interest in the population tested. Another reason is that the absolute benefit of an effective intervention tends to be greater for high-risk patients. ${ }^{6}$

No randomised controlled trials (RCTs) have been completed in asymptomatic high-risk men to

\section{Abstract}

Objectives: To apply the most recent evidence from randomised trials of prostate-specific antigen (PSA) screening and explore the potential value of risk assessments to guide the use of PSA screening in practice.

Design: A decision model that incorporated a Markov process was developed in 2012 to estimate the net benefit and cost of PSA screening versus no screening as a function of baseline risk.

Main outcome measures: Quality-adjusted life-years (QALYS) and costs.

Results: The harms of screening outweighed the benefits under a number of plausible scenarios. Conclusions were sensitive to the estimated quality-of-life impacts of prostate cancer treatment as well as the incidence of cancers not detected by screening tests (poorer prognosis) and those that were detected by screening tests (better prognosis). The base-case incremental cost effectiveness ratio of PSA screening was $\$ 168611$ per QALY for men with average risk, $\$ 73452$ per QALY for men with two times the average risk, and $\$ 22938$ per QALY for men with five times the average risk.

Conclusions: PSA screening was not found to be cost-effective for men at an average-to-high risk of prostate cancer, but may be cost-effective for men at very high risk. Inexpensive approaches for identifying men at very high risk are needed, as is further research on the size of clinical benefit of early detection in this population. The potential for the costs of risk assessment to be offset by reduced costs of PSA screening also warrants investigation.

inform the size of the possible benefits and harms of PSA screening. Observational studies of screening in higher risk groups have not provided definitive evidence about these tradeoffs. ${ }^{5}$ Statistical modelling techniques have been used to provide valuable insights about the likely impact of PSA screening on quality of life (QOL) and cost-effectiveness; 7,8 however, few studies have examined the trade-off between benefits and harms for high-risk populations., ${ }^{3,4}$ Recently updated outcome data from the largest of the PSA screening trials provide an opportunity to improve our understanding of this trade-off as a function of baseline risk through statistical modelling. ${ }^{9}$

An improved estimate of the potential net clinical impact and cost of using PSA screening in populations with varying levels of risk is particularly relevant given the discovery of molecular markers that may help classify prostate cancer risk. Risk assessment tools that include these markers appear capable of identifying men with a two to five times increased risk compared with the average. ${ }^{10-12}$ PSA screening in the subgroup of asymptomatic men at very high risk (ie, five times above the average) and those at high risk (two times above the average) may confer an appreciable net benefit in terms of quality-adjusted survival time and be cost-effective.

The aim of this study was to use the most recent evidence from RCTs of PSA screening to explore the potential value of using risk assessments to guide the use of PSA screening in practice.

\section{Methods}

A decision model that incorporated a Markov process was developed using TreeAge Pro 2009 (TreeAge Software Inc) to estimate the lifetime net benefit and cost of PSA screening versus no screening in a simulated cohort of 50-year-old men. Markov processes were used to model the experience of patients as a progression through a series of discrete health states over a time horizon divided into periods, or cycles, of equal duration. Transition probabilities determined the chance of moving from one health state to 
another at the conclusion of each cycle. Each health state was assigned a QOL utility weighting (on a scale from 0 to 1 ) and a cost for health care provision over the cycle. The expected cumulative quality-adjusted survival time and financial costs accrued over the entire process were estimated by summing the health gains and costs incurred over all cycles. The effect on the conclusions of varying the parameter estimates included in the model was explored in sensitivity analyses.

A Markov process employing a 1year cycle length was constructed to model the policy of PSA screening every 4 years against the policy of no PSA screening (Box 1). The transition probabilities used in the model are summarised in Box 2, together with the ranges applied in the sensitivity analyses.

The model made a distinction between prostate cancers that were detected by screening tests (screendetected) and those that were not detected by screening tests (nonscreen-detected). The results of the European Randomized Study of Screening for Prostate Cancer (ERSPC) trial were used to estimate the incidence of both types of prostate cancer. ${ }^{9}$ Incidence rates were age-adjusted based on Australian age-specific incidence figures. We assumed a PSA test sensitivity of $44 \%$ and specificity of $92 \%$ at a $4 \mathrm{ng} / \mathrm{mL}$ cut-point on the basis of previous estimates. ${ }^{13}$

Initial estimates of the prostate cancer mortality rate given screendetected cancer, and the corresponding rate given non-screen-detected cancer, were informed by examining the risk profile of patients in the ERSPC trial in light of published prognostic models for localised prostate cancer. ${ }^{14,15}$ Initial estimates were refined by calibrating the model against the results of the ERSPC trial. The 10-year prostate cancer mortality probability was consequently estimated to be $16 \%$ for men with nonscreen-detected cancer, and the relative risk of prostate cancer mortality for men with screen-detected prostate cancer was 0.87. Age-specific nonprostate cancer mortality rates were calculated from Australian population statistics.

Each health state was assigned an estimate of the resources required to

\section{Schema of Markov process modelling the policy of prostate-specific antigen (PSA) screening every 4 years versus the policy of no PSA screening in a simulated cohort of 50-year-old men*}

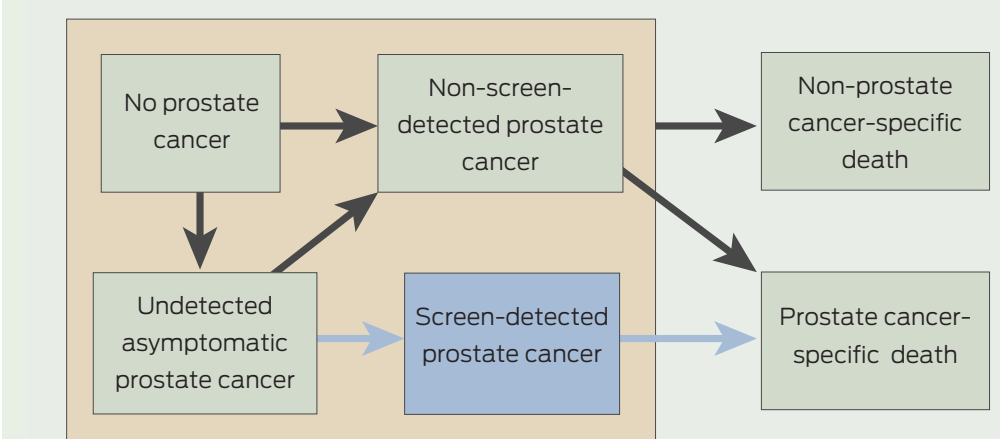

*All men in the simulated cohort entered the Markov process with no prostate cancer (the upper-left green rectangle in diagram). Given a policy of no PSA screening, and at the completion of each 1-year cycle, men in the simulated cohort could transition to any of the states represented by the green rectangles, following the direction indicated by the arrows. Given a policy of PSA screening, men in the simulated cohort could transition in accordance with any arrows (blue or black) to any health state. Under either policy, the model allowed men in the simulated cohort to remain in any of the transient states (states other than death) for more than one cycle. The likelihood of transitioning to another state at the completion of each 1-year cycle was governed by the probabilities in Box 2 .

provide appropriate health care. These costs were estimated from the perspective of the Australian health care system and included the direct health care costs associated with the natural history of prostate cancer. Box 3 summarises costs included in the model, along with the ranges that were applied in the sensitivity analyses.

The net effectiveness of each strategy (PSA screening versus no PSA screening) was quantified in terms of quality-adjusted life-years (QALYs). The average QALYs were calculated by weighting the time spent in each health state by the health-related QOL value (utility) associated with that state, where $0=$ death and $1=$ full health. We assumed that the effect of prostate cancer (and its treatment) on QOL was equivalent to a decrement of 0.05 in utility averaged over the entire survival period, with a plausible range of 0.00 to 0.10 . This range for the decrement in utility was chosen on the basis of evidence from longitudinal evaluations of QOL in prostate cancer. ${ }^{16}$ We further assumed that QOL during the 12 months before death from prostate cancer was associated with an additional 0.50 reduction in health state utility. ${ }^{17}$

A half-cycle correction was applied in the Markov process when calculating the expected outcomes of the screening and non-screening strategies, and a 5\% discount rate was applied to both future costs and benefits.
The model described above was run on three simulated cohorts of 50-yearold men: a cohort with an average level of risk of prostate cancer (ie, risk was age-adjusted but otherwise comparable to that of men enrolled in the ERSPC trial); a second cohort with high-risk of prostate cancer; and a third cohort with a very high risk of prostate cancer. The base-case prostate cancer incidence rates were increased by a factor of 2 for the highrisk simulated cohort and by a factor of 5 for the very high-risk simulated cohort.

\section{Results}

For men with an average risk, PSA screening led to an additional 2.7 quality-adjusted life-days (14.3 undiscounted) at an additional cost of \$2185, giving an incremental costeffectiveness ratio (ICER) of \$291817 per QALY gained. For the high-risk cohort, PSA screening led to an additional 8.3 quality-adjusted life-days (43.0 undiscounted) at an additional cost of \$2519, giving an ICER of \$110726 per QALY gained. For the very high-risk cohort, PSA screening led to an additional 32.9 qualityadjusted life-days (161.2 undiscounted) at an additional cost of $\$ 2755$, giving an ICER of $\$ 30572$ per QALY gained (see Box 4 for detail on the cumulative event rates).

PSA screening was associated with a net gain in QALYs under many, but 
2 Decision model: probabilities and utilities used to estimate lifetime net benefit and cost of prostate-specific antigen (PSA) screening versus no screening in simulated cohort of 50-year-old men

Range

\begin{tabular}{|c|c|c|c|c|}
\hline \multirow[b]{2}{*}{ Source variable } & \multirow[b]{2}{*}{ Base case } & & & \multirow[b]{2}{*}{ Source } \\
\hline & & Low & High & \\
\hline \multicolumn{5}{|l|}{ Proportions } \\
\hline PSA sensitivity & 0.44 & 0.33 & 0.59 & \multirow{2}{*}{$\begin{array}{c}\text { Low and high range corresponds to estimated test characteristics at } \\
\text { PSA cut-offs of } 5 \mathrm{ng} / \mathrm{mL} \text { and } 3 \mathrm{ng} / \mathrm{mL} .^{13} \text { Note: sensitivity increases } \\
\text { as a function of screening rounds (eg, sensitivity is } 82 \% \text { given } \\
\text { three rounds of screening in base case) }\end{array}$} \\
\hline PSA specificity & 0.92 & 0.87 & 0.95 & \\
\hline \multicolumn{5}{|c|}{ Annual incidence rates (per 1000) and relative risks } \\
\hline $\begin{array}{l}\text { Incidence of asymptomatic } \\
\text { (screen-detectable) cancers }\end{array}$ & 6.12 & 4.89 & 7.34 & $\begin{array}{l}\text { Screen-detected prostate cancer incidence rate in screening arm } \\
\text { of ERSPC, } 9 \text { and age-adjusted incidence rate based on } \\
\text { Australian age-specific figures. Low-high, } 80 \%-120 \%\end{array}$ \\
\hline $\begin{array}{l}\text { Incidence of non-screen-detected } \\
\text { prostate cancer given no screening }\end{array}$ & 5.65 & 4.52 & 6.78 & $\begin{array}{c}\text { Prostate cancer incidence rate in screening arm of ERSPC, }{ }^{9} \\
\text { and age-adjusted incidence rate based on Australian age-specific figures. } \\
\text { Low-high, } 80 \%-120 \%\end{array}$ \\
\hline $\begin{array}{l}\text { Relative risk of non-screen- } \\
\text { detected prostate cancer given }\end{array}$ & 0.49 & 0.40 & 0.59 & $\begin{array}{c}\text { Ratio of incidence rates for non-screen-detected cancers, given screening, } \\
\text { versus control arms of ERSPC. }{ }^{9} \text { Low-high, } 80 \%-120 \%\end{array}$ \\
\hline
\end{tabular}

screening

Incidence of prostate cancer death 17.28 (ie, 10-year 10.48 (ie, 10- 22.07 (ie, 10-year givennon-screen-detected prostate prostate cancer year prostate prostate cancer cancer $\begin{array}{cc}\text { mortality of } & \text { cancer mortality mortality of } 20 \% \text { ) }\end{array}$ $16 \%$ ) of $10 \%$ )

Relative risk of death from prostate 0.87 0.90

0.70 Initial estimates based on Gleason score profile of patients in the
screening and control arms of ERSPC, ${ }^{9}$ in light of two published
prognostic models for localised prostate cancer..$^{14,15}$ Preliminary
estimates were then refined by comparing the outcomes predicted
by the model against the rate actually observed in the ERSPC trial $^{9}$ cancer given screen-detected prostate cancer

Age-specific rates prostate cancer

\section{Quality of life}

Utility of prostate cancer (diagnosis and treatment)

Utility of health state 12 months before death from prostate cancer

0.95

0.90

0.50
0.30
1.0

0.70
ABS mortality data (adjusted to exclude prostate cancer deaths)

Effect of prostate cancer (and its treatment) on quality of life was assumed to be equivalent to a decrement of 0.05 in utility averaged over the entire survival period ${ }^{16}$

Quality of life during the 12 months before death from prostate cancer was assumed to be associated with an additional reduction of 0.50 in health state utility ${ }^{17}$

ABS = Australian Bureau of Statistics. ERSPC = European Randomized Study of Screening for Prostate Cancer. ${ }^{9}$

\section{Unit costs for health care resources included in Markov model}

\begin{tabular}{|c|c|c|c|c|}
\hline \multirow[b]{2}{*}{ Costs per patient } & \multirow[b]{2}{*}{ Base case } & \multicolumn{2}{|c|}{ Range } & \multirow[b]{2}{*}{ Source } \\
\hline & & Low & High & \\
\hline Lifetime treatment costs (excluding terminal care) & $\$ 22077$ & $\$ 17662$ & $\$ 26492$ & $18 * \dagger$ \\
\hline Confirming cancer diagnosis (one-off cost) & $\$ 571$ & $\$ 457$ & $\$ 685$ & \\
\hline Additional PSA & $\$ 37.55$ & & & MBS item 66660 \\
\hline Biopsy & $\$ 408.70$ & & & MBS item 37215 \\
\hline $2 \times$ urologist visits & $\$ 125.00$ & & & MBS items 104, 105 \\
\hline Routine PSA screening & $\$ 87.95$ & $\$ 70$ & $\$ 106$ & \\
\hline General practitioner visit (Level C) $)^{\ddagger}$ & $\$ 67.65$ & & & MBS item 36 \\
\hline Cost of test ${ }^{\xi}$ & $\$ 20.30$ & & & MBS item 66655 \\
\hline Terminal care for prostate cancer & $\$ 8458$ & $\$ 6766$ & $\$ 10150$ & $18 * 9$ \\
\hline Non-prostate cancer death & $\$ 8700$ & $\$ 6960$ & $\$ 10440$ & $19 *$ \\
\hline
\end{tabular}

AlHW = Australian Institute of Health and Welfare. MBS = Medicare Benefits Schedule. PSA = prostate-specific antigen. $*$ Inflated to reflect 2012 prices. ${ }^{20}+$ Based on AlHW estimates of the lifetime costs of prostate cancer in Australia, adjusted to reflect that non-terminal care costs represent $72.3 \%$ of lifetime costs. ${ }^{21} \neq$ Set to 0 in an additional sensitivity analysis under the assumption that PSA testing could be performed during a routine appointment scheduled for other reasons. $\oint$ PSA screening cost estimated based on fee for single quantitation and varied up to fee for quantitation of multiple fractions or derived index in sensitivity analysis. 9 Based on AlHW estimates of the lifetime costs of prostate cancer in Australia, adjusted to reflect that terminal care costs represent $27.7 \%$ of lifetime costs. ${ }^{21}$

not all, of the plausible scenarios run in the sensitivity analyses (Box 5). A threshold for effectiveness, beyond which screening was detrimental (ie, leading to a reduction in QALYs), was found in the following scenarios:

- when the utility of prostate cancer (diagnosis and treatment) was set to less than 0.93 for the average-risk cohort, and less than 0.91 for the high-risk cohort;

- for all risk cohorts when the annual reference rate of non-screen-detected prostate cancer given no screening was less than approximately $0.5 \%$;

- for average- and high-risk cohorts when the annual reference rate of asymptomatic (screen-detectable) prostate cancer was greater than approximately $0.7 \%$; and

- for the average-risk cohort when the annual rate of annual rate of death from prostate cancer given nonscreen-detected prostate cancer was less than $1.14 \%$.

Sensitivity analyses on financial costs showed PSA screening to always be more costly than no screening. The most influential parameters on the incremental cost of screening included the cost of the PSA testing, the cost of treatment for prostate cancer, the annual rate of non-screen-detected prostate cancer given no screening, the annual rate of screen-detectable prostate cancer and the relative risk of non-screendetected prostate cancer given screening.

\section{Discussion}

PSA screening was associated with a net gain in QALYs for all patient risk groups under many, but not all, of the plausible scenarios explored in our model. The harms of screening outweighed the benefits when the inci- 


\begin{tabular}{|c|c|c|c|c|c|c|}
\hline \multirow[b]{2}{*}{ Outcomes } & \multicolumn{2}{|c|}{ Average-risk cohort } & \multicolumn{2}{|c|}{ High-risk cohort } & \multicolumn{2}{|c|}{ Very high-risk cohort } \\
\hline & No screening & PSA screening & No screening & PSA screening & No screening & PSA screening \\
\hline \multicolumn{7}{|l|}{ Lifelong total (10-year projected outcomes) } \\
\hline PSA screening tests performed & & $7.66(2.93)$ & & $7.03(2.92)$ & & $5.72(2.89)$ \\
\hline False-positive result of PSA screening tests & & $0.58(0.23)$ & & $0.51(0.22)$ & & $0.37(0.21)$ \\
\hline \multicolumn{7}{|c|}{ Cumulative incidence per 1000, lifelong total (10-year projected outcomes) } \\
\hline Screen-detected prostate cancer & na & $182.95(23.91)$ & na & $306.65(47.42)$ & na & $492.81(113.08)$ \\
\hline Non-screen-detected prostate cancer & $194.4(28.15)$ & $90.53(14.1)$ & $344.5(56.04)$ & $154(27.4)$ & $627.03(135.74)$ & $254.63(65.44)$ \\
\hline Death from prostate cancer & $45.35(1.72)$ & $37.55(1.3)$ & $82.16(3.43)$ & $65.83(2.61)$ & $163.38(8.62)$ & $117.63(6.42)$ \\
\hline Death from other causes & $954.63(41.93)$ & $962.43(42.08)$ & $917.83(41.74)$ & $934.16(41.69)$ & $836.62(41.45)$ & 882.35 (41.57) \\
\hline
\end{tabular}

dence of non-screen-detected (poorer prognosis) cancers was varied towards the lower end of the range tested. For average-to-high-risk groups, PSA screening was also estimated to be harmful when the incidence of screendetected (better prognosis) cancers was estimated at the higher end of the range tested, and when the QOL impacts of prostate cancer treatment exceeded a relatively modest threshold.

PSA screening was not found to be cost-effective for men with an average risk of prostate cancer. We estimated that for a 50-year-old man with average risk, PSA screening would lead to an average additional $\$ 2185$ per individual screened, with negligible increase in qualityadjusted survival. The cost-effectiveness of screening remained unfavourable for the high-risk cohort at \$110 726 per QALY gained, but was considerably more attractive for the very high-risk group at $\$ 30572$ per QALY gained. While there is currently no explicit threshold for an ICER at which an intervention is considered cost-effective for funding purposes in Australia, recent MSAC assessments have not recommended public reimbursement for preventative interventions with ICERs well over this amount.

The prostate cancer treatment costs we projected from past data may be an underestimate of future costs, assuming that access to a growing number of newer and more costly pharmaceutical therapies increases over time. The upper limit of the treatment cost estimate used in our sensitivity analysis was chosen to explore the consequence of this uncertainty, and we found that conclusions were unchanged.

5 Results of sensitivity analyses showing difference in quality-adjusted life-years after each of the parameters shown in Box 2 was varied through its plausible range*

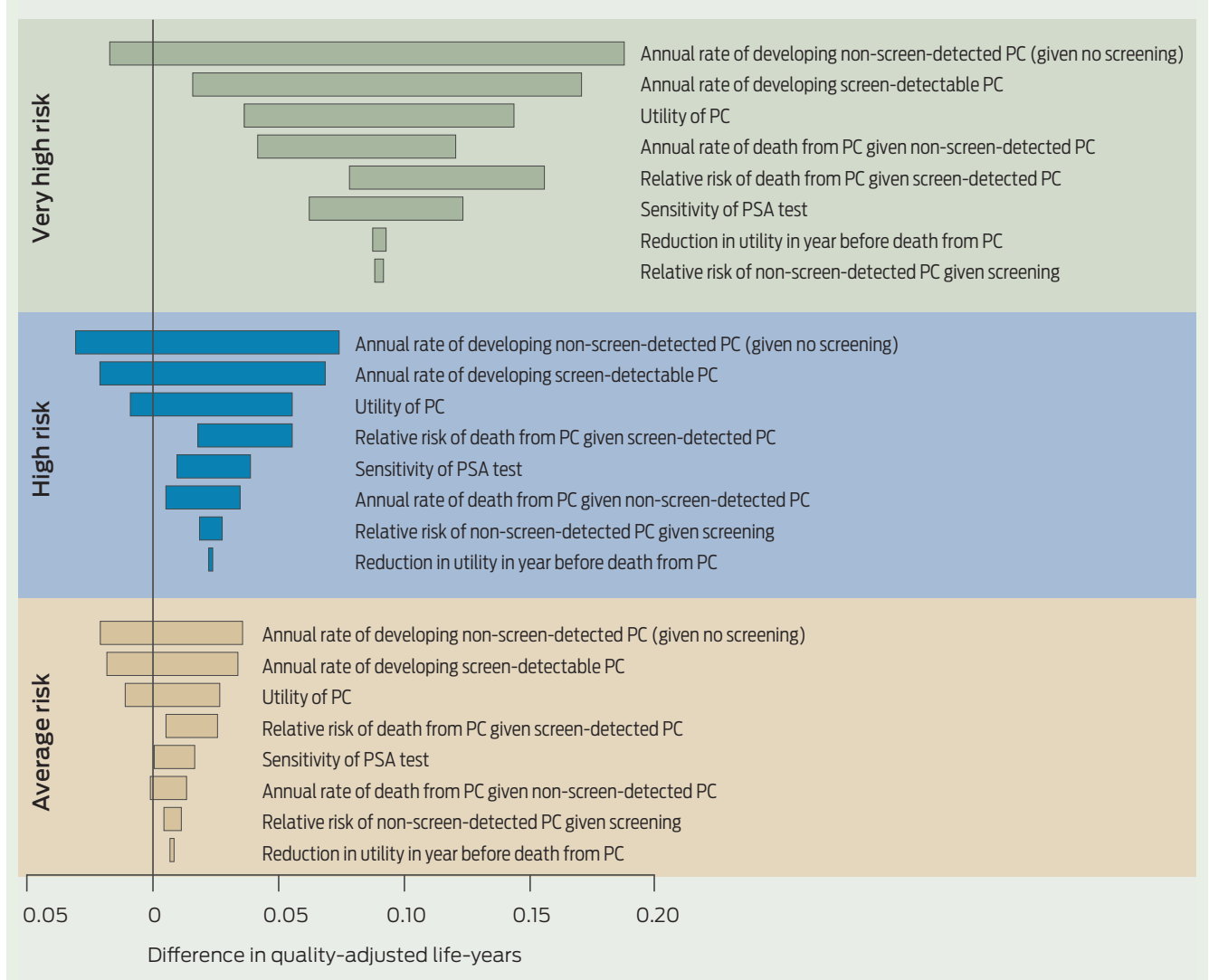

$\mathrm{PC}=$ prostate cancer. $\mathrm{PSA}=$ prostate-specific antigen. $*$ Each parameter in Box 2 was varied through its plausible range in a series of one-way sensitivity analyses for each of the three simulated cohorts. The results are presented in the form of a tornado diagram with horizontal bars showing how the net effectiveness of PSA screening changes as each parameter was varied through its plausible range. Values to the right of the vertical line represent a result indicating a net benefit and those to the left represent a result indicating a net harm.

Our findings have direct implications for the strategy of providing personalised risk information to the subset of men who are actively seeking PSA screening. ${ }^{2}$ Assessing patient family history to identify men at 2- to 3.5-fold increased risk of prostate cancer can be used to identify a group of men for whom PSA screening will provide a greater net clinical benefit; ${ }^{22}$ however, our find- ings suggest that these benefits are not large enough to produce a favourable cost-effectiveness ratio.

The use of molecular markers to identify patients at very high risk for more targeted screening is promising. However, critical factors include the prevalence of very high risk markers, the cost of risk assessment and the potential for cost offsets if men identified at average risk are less likely to 
favour screening. Only a relatively small proportion of the population of 50 -year-old men is likely to be identifiable at very high risk (ie, $\geqslant$ five times the average risk), based on recent studies. The prevalence of high-risk mutations in the BRCA1 and BRCA2 genes, for example, has been estimated to be only around $0.5 \%$ in the general population. ${ }^{23}$ According to a five-single-nucleotide polymorphisms (SNPs) prostate cancer risk factor model, ${ }^{11}$ only $0.3 \%$ of men in the control group fell into the very high-risk category (ie, $\geqslant$ five times the average risk), while in a 25-SNP model, not even those at the upper 99th percentile of SNP profile distribution reached this level. ${ }^{12}$

The costs of testing across the general population to identify a relatively small group of very high-risk men (comprising less than 1\% of the general population) will be critical determinants of cost-effectiveness. The current costs of genetic tests such as that for BRCA1 and BRCA2 are relatively high, and thus populationbased testing could not be justified to guide PSA screening.

The cost of providing a risk assessment has the potential to be offset by cost savings if those found to be at moderate levels of risk subsequently choose not to pursue routine PSA screening. The degree of cost offset would be a function of, among other things, the accuracy of the risk assessment tool, the distribution of risk profiles across the target population and the ability of information from a personalised risk assessment to influence patient preferences for PSA screening. Further empirical research is needed on the potential of these factors to offset the cost of personalised risk assessment.

Our analysis uses the most recent evidence from randomised trials of PSA screening to provide a better understanding of the potential impacts on survival, QOL and costs of PSA screening as a function of personal risk. The results of our study are limited by the rigour of the available evidence from randomised trials and do not obviate the need for longer-term randomised trials in higher risk men, ${ }^{24}$ or for data from ongoing observational studies such as IMPACT (Identification of Men with a Genetic Predisposition to Prostate Cancer: Targeted Screening in BRCA1/2 Mutation Carriers and Controls), which is evaluating annual PSA screening in men with a BRCA1 or BRCA2 mutation. ${ }^{5}$ Although our model suggests that there is potential for PSA screening in very high-risk cohorts, it may underestimate the harms associated with screening if the effect of false-positive PSA test results and biopsies on QOL is not short-lived. Further data are required on the impact of false-positive results and associated biopsies on QOL. Our model does, however, formally synthesise the current available evidence on PSA screening in response to the important clinical question of its potential value in higher risk populations, while incorporating the QOL impact of diagnosis and treatment as well as financial costs in the Australian context.

A major issue applicable to all evaluations of PSA screening is the lack of a reliable test for determining the subset of screen-detected prostate cancers that warrant radical treatment. A test that could reliably distinguish prostate cancers that are both amenable to radical local treatment and likely to be morbid and life-threatening without local treatment would have a favourable impact on the effectiveness and cost-effectiveness of screening.

In conclusion, PSA screening was not found to be cost-effective for men at an average-to-high risk of prostate cancer, but may be cost-effective for men at very high risk. Inexpensive approaches for identifying men at very high risk are needed, as is further research on the size of clinical benefit of early detection in this population and the potential for costs of riskassessment to be offset by reduced costs of PSA screening.

Competing interests: No relevant disclosures.

Received 29 Oct 2012, accepted 20 Mar 2013.

1 Djulbegovic M, Neuberger MM, Dahm P. Prostatecancer mortality after PSA screening. N Engl J Med 2012; 366: 2228-2229; author reply 30-1.

2 Royal Australian College of General Practitioners. Guidelines for preventive activities in general practice. Melbourne: RACGP, 2012.

3 Pashayan N, Duffy SW, Chowdhury S, et al. Polygenic susceptibility to prostate and breast cancer: implications for personalised screening. $\mathrm{Br}$ Cancer 2011; 104: 1656-1663.

4 Howard K, Barratt A, Mann GJ, Patel Ml. A model of prostate-specific antigen screening outcomes for low- to high-risk men: information to support informed choices. Arch Intern Med 2009; 169: 1603-1610.

5 Mitra AV, Bancroft EK, Barbachano Y, et al. Targeted prostate cancer screening in men with mutations in $B R C A l$ and $B R C A 2$ detects aggressive prostate cancer: preliminary analysis of the results of the IMPACT study. BJU Int 2011; 107: 28-39.

6 Glasziou PP, Irwig LM. An evidence based approach to individualising treatment. $B M J$ 1995; 311 : 1356-1359.

7 HeijnsdijkEA, Wever EM, Auvinen A, et al. Qualityof-life effects of prostate-specific antigen screening. N Engl J Med 2012; 367: 595-605.

8 Shteynshlyuger A, Andriole GL. Cost-effectiveness of prostate specific antigen screening in the United States: extrapolating from the European study of screening for prostate cancer. J Urol 2011; 185: 828-832.

9 Schröder FH, Hugosson J, Roobol MJ, et al. Prostate-cancer mortality at 11 years of follow-up. N Engl J Med 2012; 366: 981-990.

10 LindströmS, Schumacher FR, Cox D, et al. Common genetic variants in prostate cancer risk prediction ${ }^{-}$ results from the $\mathrm{NCI}$ Breast and Prostate Cancer Cohort Consortium (BPC3). Cancer Epidemiol Biomarkers Prev 2012; 21: 437-444.

11 Zheng SL, Sun J, Wiklund F, et al. Cumulative association of five genetic variants with prostate cancer. N Engl J Med 2008; 358: 910-919.

12 Macinnis RJ, Antoniou AC, Eeles RA, et al. A risk prediction algorithm based on family history and common genetic variants: application to prostate cancer with potential clinical impact. Genet Epidemiol 2011; 35: 549-556.

13 Holmström B, Johansson M, Bergh A, et al. Prostate specific antigen for early detection of prostate cancer: longitudinal study. BMJ 2009; 339: b3537.

14 Eggener SE, Scardino PT, Walsh PC, et al. Predicting 15-year prostate cancer specific mortality after radical prostatectomy. J Urol 2011; 185: 869-875.

15 Albertsen PC, Hanley JA, Fine J. 20-year outcomes following conservative management of clinically localized prostate cancer. JAMA 2005; 293 : 2095-2101.

16 Smith DP, King MT, Egger S, et al. Quality of life three years after diagnosis of localised prostate cancer: population based cohort study. BMJ 2009; 339: b4817.

17 Stewart ST, Lenert L, Bhatnagar V, Kaplan RM. Utilities for prostate cancer health states in men aged 60 and older. Med Care 2005; 43: 347-355.

18 Australian Institute of Health and Welfare. Health system expenditures on cancer and other neoplasms in Australia 2000-01. Canberra: AlHW, 2005. (AlHW Cat. No. HWE 29; Health and Welfare Expenditure Series No. 22.) http://www.aihw.gov. au/publication-detail/?id=6442467719 (accessed May 2013).

19 Seshamani M, Gray AM. A longitudinal study of the effects of age and time to death on hospital costs. J Health Econ 2004; 23: 217-235.

20 Australian Bureau of Statistics. Consumer price index, Australia, Jun 2012. Canberra: ABS, 2012 (ABS Cat. No. 6461.0.) http://www.abs.gov.au/ (accessed May 2013).

21 Stone CA, May FW, Pinnock CB, et al. Prostate cancer, the PSA test and academic detailing in Australian general practice: an economic evaluation. Aust N Z J Public Health 2005; 29: 349-357.

22 Johns LE, Houlston RS. A systematic review and meta-analysis of familial prostate cancer risk. $B J U$ Int 2003; 91: 789-794.

23 Risch HA, McLaughlin JR, Cole DE, et al. Population $B R C A 1$ and BRCA2 mutation frequencies and cancer penetrances: a kin-cohort study in Ontario, Canada. J Natl Cancer Inst 2006; 98: 1694-1706.

24 Miller AB. New data on prostate-cancer mortality after PSA screening. N Engl J Med 2012; 366: 1047-1048. 